\title{
Candida glabrata fungaemia: the importance of anaerobic blood culture
}

We read with great interest the report by Foster et al. (2007), who described the rapid detection of Candida glabrata from BACTEC 9240 anaerobic bottles.

We retrospectively reviewed the microbiology laboratory log of all positive blood cultures from 2003 to November 2007 in our institution. Our laboratory uses the BacT/ALERT (bioMérieux) automated blood culture system. Three types of blood culture bottles are routinely used for blood culture: aerobic bottles (SA; without charcoal), anaerobic bottles (SN; without charcoal) and Paediatric aerobic bottles (PF; supplemented with charcoal). All blood cultures are incubated for 5 days before discard. In our review, a total of 22 separate cases of fungaemia caused by C. glabrata were identified during this period. Five of the isolates $(22.7 \%)$ were recovered only from anaerobic bottles and not detected from aerobic bottles. Another one showed a longer time to detection (TTD) from the aerobic bottle than from the anaerobic bottle (70.3 h vs $12 \mathrm{~h}$, respectively).

We further analysed the blood culture system using the simulation method described by Foster et al. (2007). Briefly, C. glabrata ATCC 90030 and four clinical blood culture isolates were used. Inocula were prepared at $10^{2}$ and $10^{3}$ cells $\mathrm{ml}^{-1}$ for each strain. One millilitre of each Candida suspension was added to blood culture bottles inoculated with waste whole blood from the hospital blood bank service. Three types of bottles, aerobic bottles (SA; without charcoal), anaerobic bottles (SN; without charcoal) and Paediatric aerobic bottles (PF; supplemented with charcoal), were analysed in this study. All bottles were incubated in the BacT/ALERT system, with the TTD recorded automatically. Terminal subculture for all bottles was performed to ensure purity of the culture.

The results from the simulated C. glabrata fungaemia are shown in Table 1. The anaerobic bottles consistently revealed a significantly shorter TTD when compared with both types of aerobic bottles $(P$ $<0.001$ ) for both $10^{2}$ and $10^{3}$ c.f.u. $\mathrm{ml}^{-1}$ inocula. Moreover, the Paediatric aerobic bottles (PF; supplemented with charcoal) were the last to flag positive when compared to the other two types of bottle $(P<0.001)$. Horvath et al. $(2007)$ also showed a longer TTD with FA bottles (aerobic bottles supplemented with charcoal) in the BacT/ALERT 3D system. This, together with our results, suggests that for automated blood culture systems (BACTEC 9240, BacT/ALERT and BacT/ ALERT 3D), the use of anaerobic bottles significantly shortens the TTD for $C$. glabrata fungaemia. In addition, the use of blood culture bottles supplemented with charcoal resulted in further prolonged TTD.

In paediatric patients, the volume of blood obtained for investigation can be very limited. As anaerobes are rarely a major cause of sepsis in children, it had been advocated to inoculate the whole volume into a single paediatric blood culture bottle (Dunne et al., 1994; Freedman \&
Roosevelt, 2004). However, as our results have shown, such practice may not only prolong the time required to detect $C$. glabrata, but may also miss out a portion of cases. Therefore, to balance the benefits and cost of blood culturing, in selected cases where the suspicion of candidaemia is high, the anaerobic blood cultures should not be omitted.

\section{Mamie Hui, Wing-Han Ho, Wai-Ho Lam, Chi-Sing Poon, Kai-Cheong Chu and Chiu-Yeung Chan}

Department of Microbiology, The Chinese University of Hong Kong, The Prince of Wales Hospital, Hong Kong SAR

Correspondence: Mamie Hui (mamiehui@cuhk.edu.hk)

Dunne, W. M., Jr, Tillman, J. \& Havens, P. L. (1994). Assessing the need for anaerobic medium for the recovery of clinically significant blood culture isolates in children. Pediatr Infect Dis J 13, 203-206.

Foster, N., Symes, C., Barton, R. \& Hobson, R. (2007). Rapid identification of Candida glabrata in Candida bloodstream infections. J Med Microbiol 56, 1639-1643.

Freedman, S. B. \& Roosevelt, G. E. (2004). Utility of anaerobic blood cultures in a pediatric emergency department. Pediatr Emerg Care 20, 433-436.

Horvath, L. L., George, B. J. \& Hospenthal, D. R. (2007). Detection of fifteen species of Candida in an automated blood culture system. J Clin Microbiol 45, 3062-3064.

Table 1. Time to detection ( $h$, mean \pm standard deviation) in the BacT/ALERT system with the indicated inocula of Candida glabrata

\begin{tabular}{|lcc|}
\hline Bottle type & \multicolumn{2}{c|}{ Inoculum size (c.f.u. $\mathbf{~ m l}^{\mathbf{- 1}}$ ) } \\
\cline { 2 - 3 } & $\mathbf{1 0 0}$ & $\mathbf{1 0 0 0}$ \\
\hline Anaerobic bottle (SN; without charcoal) & $14.8 \pm 1.0$ & $12.2 \pm 1.0$ \\
Aerobic bottle (SA; without charcoal) & $29.9 \pm 9.0$ & $20.2 \pm 6.4$ \\
Paediatric bottle with charcoal (PF) & $45.7 \pm 9.5$ & $33.2 \pm 7.1$ \\
\hline
\end{tabular}

